\title{
Translation and validation of the Dutch language version of the CDC Symptom Inventory for assessment of Chronic Fatigue Syndrome (CFS) Ruud CW Vermeulen*
}

Address: CFS and Pain Research Center Amsterdam, Amsterdam, The Netherlands

Email: Ruud CW Vermeulen* - rv@cvscentrum.nl

* Corresponding author

Published: 18 October 2006

Population Health Metrics 2006, 4:12 doi:10.1 I86/1478-7954-4-12

This article is available from: http://www.pophealthmetrics.com/content/4/I/12

(c) 2006 Vermeulen; licensee BioMed Central Ltd.

This is an Open Access article distributed under the terms of the Creative Commons Attribution License (http://creativecommons.org/licenses/by/2.0), which permits unrestricted use, distribution, and reproduction in any medium, provided the original work is properly cited.
Received: 25 June 2006

Accepted: 18 October 2006

\begin{abstract}
Background: In a study by Wagner et al., the CDC Symptom Inventory was validated in a population selected from the inhabitants of a city in the USA, and proofed reliable for the assessment of the accompanying symptoms of CFS. The Dutch translation of the CDC Symptom Inventory is compared to the original and the psychometric properties are presented for patients in a tertiary care setting.
\end{abstract}

Methods: One hundred thirty-nine consecutive patients who visited the CFS Center Amsterdam for the first time were asked to complete the CDC Symptom Inventory in the Dutch Language Version (DLV) together with the usual set of questionnaires. Sixty-one patients had Chronic Fatigue (CF) and 78 patients fulfilled the criteria for CFS. Forty-three healthy accompanying persons completed the CDC Symptom Inventory DLV, the Physical Functioning scale of the Medical Outcome Survey Short Form-36 DLV, and the Fatigue and Concentration scales of the Checklist Individual Strength (CIS-20).

Results: The healthy controls group contained fewer women and was overall older than the patient groups. The influence of gender on the CDC Symptom Inventory DLV was significant but the effect of age was not. The Dutch version had a good internal consistency and convergent validity. The results were comparable to the original English version, but the sex-related difference needs further study.

Conclusion: The Dutch version of the CDC Symptom Inventory is a reliable tool for the assessment of the secondary criteria for CFS. The results show that it is comparable to the outcome of studies in English speaking countries.

\section{Background}

Chronic Fatigue Syndrome (CFS) is a disabling state that was defined by a working group in 1994 [1]. The main components of the definition are fatigue that is not related to exercise and not relieved by rest, and eight accompany- ing symptoms, of which four must be present. The CFS is incapacitating, with a serious reduction in daily activity.

Several self-rating scales for the presence and severity of fatigue were developed. Of these, the Multidimensional Fatigue Inventory (MFI-20) [2] and the Checklist Individ- 
ual Strength (CIS-20) [3] were selected by an international CFS study group [4]. The same group advised the Medical Outcomes Survey Short-Form-36 (SF-36) as the tool for the assessment of functional impairment. For the presence of the accompanying symptoms of CFS, a symptom checklist developed by the Centers for Disease Control and Prevention was suggested. The MFI-20 and the CIS-20 were developed in the Dutch language and validated. The SF-36 was translated and validated [5]. The CDC Symptom Inventory was validated for the English-speaking countries [6]. The translation in Dutch was considered necessary for comparison of data in CFS research in the Netherlands and other countries. The objective of the present study was to translate the CDC Symptom Inventory and to validate it for the Dutch speaking population.

\section{Methods}

The participants in the study were all patients who attended the CFS Center Amsterdam for the first time for diagnosis and treatment of chronic fatigue from August 2005 to August 2006 and their healthy accompanying persons. No investigations were added to the standard diagnostic protocol for new patients in the Center and the accompanying persons were asked to complete the questionnaires only. Accompanying persons who reported fatigue of one month or more, or were ever identified with medical or psychiatric conditions exclusionary for CFS were excluded from the study. All participants gave informed consent for the use of their data for this study.

The CIS-20 is a 20-item self-report instrument that measures 4 dimensions of fatigue: fatigue, concentration, impaired motivation and impaired activity. For clinical assessment of fatigue we used the fatigue and concentration subscales. The CDC Symptom Inventory DLV was used for the assessment of the presence of additional symptoms and their severity. Symptoms were rated as suggested by Wagner et al. [6]. We calculated the CDC Symptom Inventory DLV Total Score, the CDC Symptom Inventory DLV Short Form, the CDC Symptom Inventory DLV Case Definition Score and the CDC Symptom Inventory DLV Other Symptoms score as indicated by the authors. The severity of physical impairment was measured with the physical functioning subscale of the SF-36. All healthy controls completed a list of questions about health, medical interventions in the past and drug use. A physical checkup and laboratory data according to the recommendations of Fukuda et al. [1] were obtained from all patients.

\section{Translation}

The English version of the CDC Symptom Inventory was translated into Dutch by a native Dutch speaker fluent in English. The translation was presented to 4 native Dutch speakers for problems in acceptance and comprehension of the questionnaire content or the phrasing. The provisional Dutch version was translated back into English by a native English speaker fluent in Dutch.

\section{Statistical analysis}

We evaluated the internal consistency of the CDC Symptom Inventory DLV by performing a reliability analysis based on the model of averaging the inter-item correlation.

In the male and female groups the convergent validity between the CDC Symptom Inventory DLV, the CIS-20 and the physical score of the SF-36 was tested by the calculation of Pearson's correlation coefficient. Construct validity by one-way of variance analysis and Bonferroni post-hoc group comparisons were used to compare the CDC Symptom Inventory DLV scores, the CIS-20 scores and the SF-36 score across the three groups. To determine whether there were differences between CDC groups, a series of multivariate analyses of covariance was conducted, with physical impairment, fatigue or concentration as dependant variables and with gender and age as covariates.

All statistical analyses were carried out using the Statistical Package for the Social Sciences (SPSS version 14.0).

\section{Results}

Forty-three healthy controls completed the questionnaires. Sixty-one patients fulfilled the Fukuda criteria for chronic fatigue (CF-group) and 78 patients those for chronic fatigue syndrome (CFS-group) (Table 1). The difference between men and women was significant for all scores (Table 2) (Student's $t$-test $P<0.001$ ). The differences were partially caused by the age distributions that were not the same for men and women in the Control, CF and CFS groups. The difference of the age distribution between the 3 groups was significant for men and women together (Jonckheere-Terpstra Test: $P=0.013$ ). When tested separately the age differences between the 3 groups were neither different for women (Jonckheere-Terpstra Test: $P=0.287$ ) nor for men (Jonckheere-Terpstra Test: $P$ $=0.177)$. Analysis of covariance, controlling for age and

Table I: Characteristics by subject classification $(n=182)$.

\begin{tabular}{lcccc}
\hline Classification & \multicolumn{2}{c}{ Number } & \multicolumn{2}{c}{ Age (Mean \pm SD) } \\
\hline & Female & Male & Female & Male \\
\hline CFS & 61 & 17 & $39(13)$ & $44(12)$ \\
CF & 47 & 14 & $37(12)$ & $42(10)$ \\
Controls & 19 & 24 & $47(16)$ & $48(12)$ \\
\hline
\end{tabular}

CFS: patients with chronic fatigue syndrome, CF: patients with chronic fatigue, not meeting the criteria for CFS, and healthy controls without fatigue complaints. 
Table 2: Descriptive data of the CDC Symptom Inventory DLV, Medical outcomes Survey Short-Form 36 and Checklist Individual Health scores.

\begin{tabular}{lcccc}
\hline CDC Symptom Inventory Scores & Mean & SD & Min & Max \\
\hline Female $(\mathrm{n}=127)$ & & & & \\
Total & 83.74 & 45.15 & 0 & 201 \\
Short Form & 45.78 & 25.13 & 0 & 96 \\
CDC Case Definition & 47.61 & 24.89 & 0 & 104 \\
Other Symptoms & 36.07 & 24.02 & 0 & 105 \\
SF-36 Physical & 57.13 & 25.21 & 0 & 100 \\
CIS Fatigue & 47.60 & 12.00 & 8 & 56 \\
CIS Concentration & 25.40 & 8.65 & 5 & 35 \\
Male (n=55) & & & & \\
Total & 48.79 & 48.21 & 0 & 158 \\
Short Form & 29.89 & 29.70 & 0 & 82 \\
CDC Case Definition & 26.96 & 25.71 & 0 & 85 \\
Other Symptoms & 21.79 & 24.58 & 0 & 92 \\
SF-36 Physical & 74.73 & 25.77 & 5 & 100 \\
CIS Fatigue & 34.94 & 18.90 & 8 & 56 \\
CIS Concentration & 18.09 & 10.59 & 5 & 35 \\
& & & & \\
\hline
\end{tabular}

gender, demonstrated significant differences between the three groups $[\Lambda=.165, F(6,174)=84.7, p<.000]$. The analysis for men and women was done separately.

\section{Reliability analysis}

Reliability analyses showed good internal consistency for the CDC Symptom Inventory Total score with a Cronbach's alpha coefficient of 0.86 for women and 0.91 for men. Cronbach's alpha coefficients were 0.85 (women) and 0.91 (men) for the Symptom Inventory Short Form, 0.79 and 0.85 for the Symptom Inventory Case Definition score, and 0.75 and 0.82 for the Symptom Inventory Other Symptoms score.

Table 2 shows the descriptive data for the Total, Short Form, Case Definition and the Other Symptoms scores. Table 3 shows the corrected item-total correlations (product terms) of the symptoms for the Total score, the Short Form and the Case Definition.

\section{Validity}

Convergent validity of the CDC Symptom Inventory

The Pearson's correlation coefficient indicated a good convergent validity of the CDC Total, Case Definition and Short Form scores as determined by correlations with the CIS and the SF-36 in men and women (Table 4). Severity of fatigue coincided with severity of accompanying symptoms and physical impairment.

Table 3: Corrected item to total correlations for the CDC Symptom Inventory DLV Total score, the Symptom Inventory Short Form score and the Symptom Inventory Case Definition score.

\begin{tabular}{|c|c|c|c|c|c|c|}
\hline \multirow[t]{3}{*}{ Symptom } & \multicolumn{6}{|c|}{ Corrected item total correlations } \\
\hline & \multicolumn{2}{|c|}{ Total Score } & \multicolumn{2}{|c|}{ Short Form } & \multicolumn{2}{|c|}{ Case Definition } \\
\hline & Female & Male & Female & Male & Female & Male \\
\hline Sore throat & .40 & .28 & & & .34 & .30 \\
\hline Tender nodes & .42 & .24 & & & .37 & .22 \\
\hline Diarrhea & .40 & .47 & & & & \\
\hline Unusual fatigue after exertion & .66 & .82 & .69 & .86 & .61 & .84 \\
\hline Muscle aches & .48 & .63 & .44 & .57 & .59 & .67 \\
\hline Joint pain & .38 & .50 & & & .43 & .52 \\
\hline Feverishness & .35 & .27 & & & & \\
\hline Chills & .36 & .60 & & & & \\
\hline Unrefreshing sleep & .69 & .83 & .75 & .87 & .61 & .82 \\
\hline Sleeping problems & .64 & .72 & .66 & .78 & & \\
\hline Headaches & .36 & .64 & & & .41 & .62 \\
\hline Memory problems & .56 & .66 & .56 & .64 & & \\
\hline Concentration & .66 & .86 & .68 & .89 & .57 & .78 \\
\hline Nausea & .49 & .42 & & & & \\
\hline Stomach pain & .45 & .47 & & & & \\
\hline Sinus problems & .27 & .40 & & & & \\
\hline Shortness of breath & .38 & .41 & & & & \\
\hline Sensitivity to light & .40 & .60 & & & & \\
\hline Depression & .41 & .56 & & & & \\
\hline
\end{tabular}


Table 4: Pearson's correlation matrix of CDC Symptom Inventory DLV, CIS-20 and SF-36 scores for women and men.

\begin{tabular}{|c|c|c|c|c|c|c|}
\hline \multirow{2}{*}{ Questionnaires } & \multicolumn{2}{|c|}{ Total score } & \multicolumn{2}{|c|}{ Short Form } & \multicolumn{2}{|c|}{ Case Definition } \\
\hline & & & & & & \\
\hline Women $(n=127)$ & $r$ & $P$ & $r$ & $P$ & $r$ & $P$ \\
\hline \multicolumn{7}{|l|}{$\mathrm{CIS}-20$} \\
\hline Fatigue & .64 & $<.001$ & 69 & $<.001$ & .65 & $<.001$ \\
\hline Concentration & .65 & $<.001$ & .69 & $<.001$ & .67 & $<.001$ \\
\hline \multicolumn{7}{|l|}{ SF-36 } \\
\hline Physical functioning & -.68 & $<.001$ & -.64 & $<.001$ & -.64 & $<.001$ \\
\hline \multicolumn{7}{|c|}{ CDC Symptom Inventory DLV } \\
\hline Total & & & .92 & $<.001$ & .93 & $<.001$ \\
\hline Short Form & .92 & $<.001$ & & & .90 & $<.001$ \\
\hline \multicolumn{7}{|l|}{$\operatorname{Men}(n=55)$} \\
\hline \multicolumn{7}{|l|}{ CIS-20 } \\
\hline Fatigue & .81 & $<.001$ & .82 & $<.001$ & .84 & $<.001$ \\
\hline Concentration & .79 & $<.001$ & .84 & $<.001$ & .79 & $<.001$ \\
\hline \multicolumn{7}{|l|}{ SF-36 } \\
\hline Physical functioning & -.84 & $<.001$ & -.80 & $<.001$ & -.85 & $<.001$ \\
\hline \multicolumn{7}{|c|}{ CDC Symptom Inventory DLV } \\
\hline Total & & & .96 & $<.001$ & .96 & $<.001$ \\
\hline Short Form & .96 & $<.001$ & & & .95 & $<.001$ \\
\hline
\end{tabular}

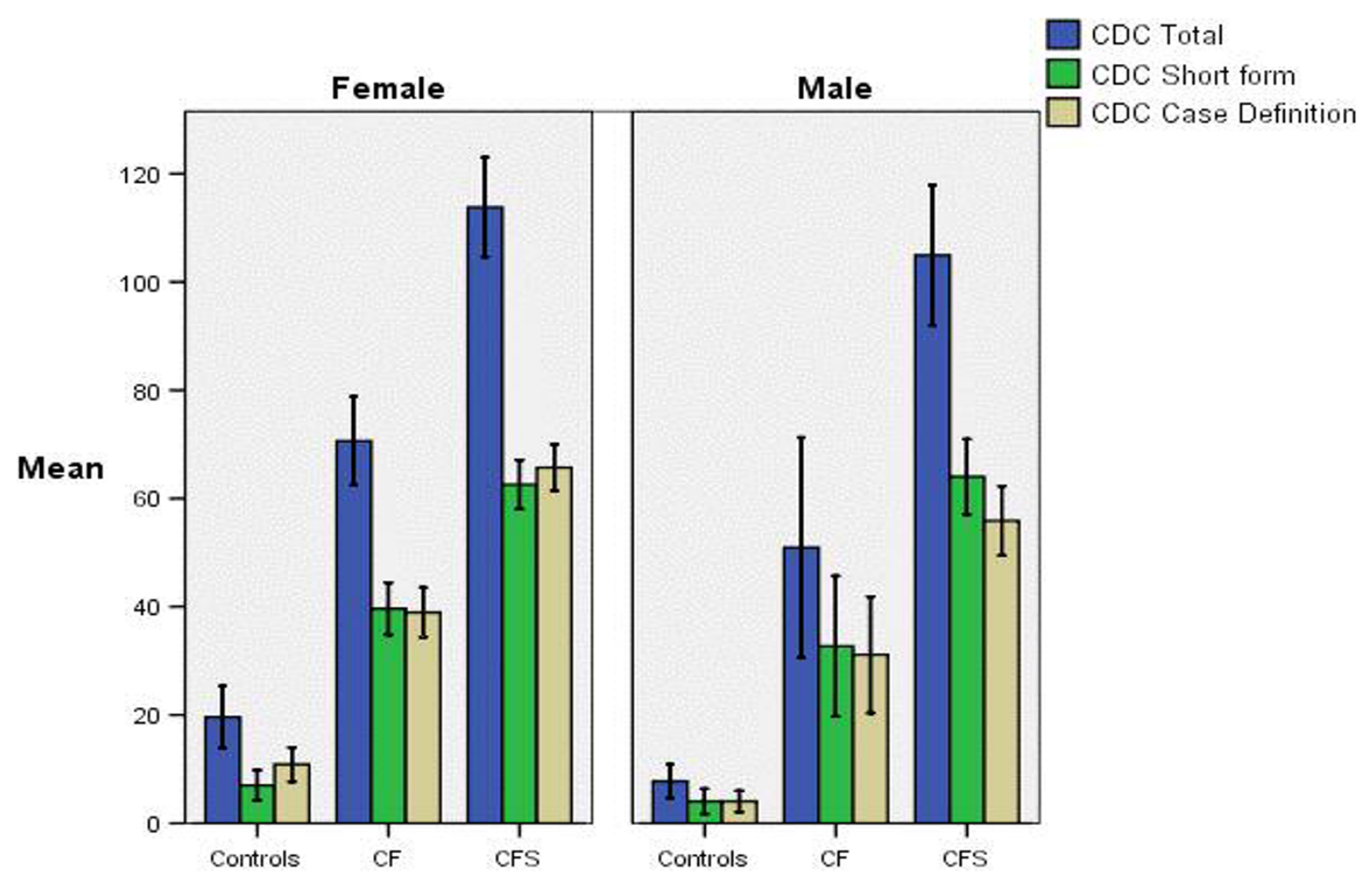

Figure I

Total, Short Form and Case Definition score of the CDC Symptom Inventory - DLV for healthy controls, Chronic Fatigue patients who did not fulfill the criteria for CFS (CF) and patients with Chronic Fatigue Syndrome (CFS). Error bars indicate Standard Error of the Mean. 


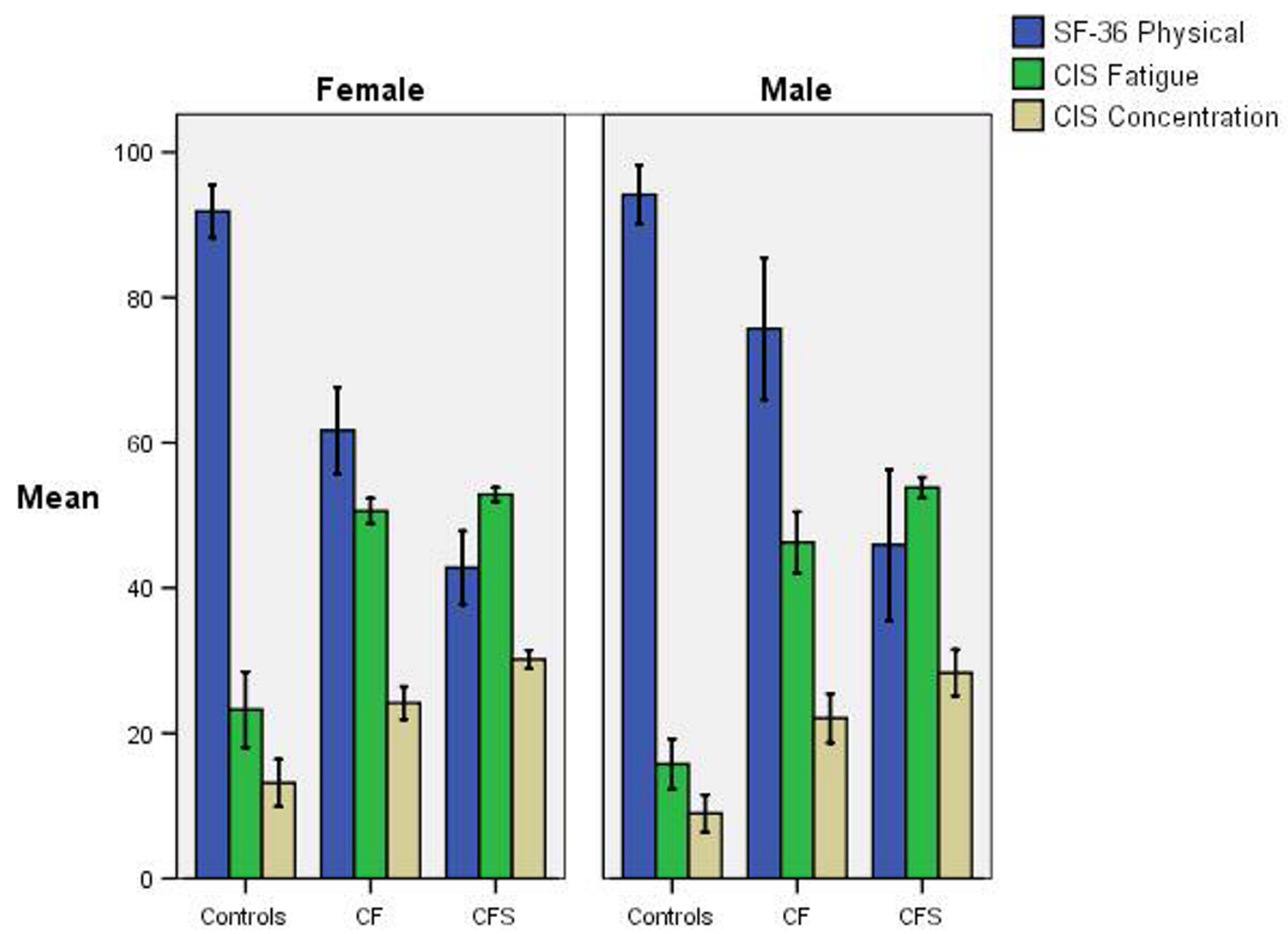

Figure 2

SF-36 Physical Functioning, CIS Fatigue and CIS Concentration scores for healthy controls, Chronic Fatigue patients who did not fulfill the criteria for CFS (CF) and patients with Chronic Fatigue Syndrome (CFS). Error bars indicate Standard Error of the Mean.

\section{Construct validity}

The Bonferroni post-hoc comparisons between healthy controls, chronic fatigue and chronic fatigue syndrome patients showed significant mean differences related to CDC Symptom Inventory scores in men and women (Figures 1 and 2) (Bonferroni post-hoc test; $P<0.001$ ).

\section{Discussion}

This evaluation of the clinical application of the Dutch translation of the CDC Symptom Inventory shows that it is a reliable tool for the assessment of CFS.

The patients differed in some respects from the population studied by Wagner et al. [6]. We analyzed consecutive patients who attended a tertiary care setting during one year and their healthy accompanying relatives and friends. The majority of CFS patients were female, the accompany- ing friends were male, and the relatives were most often parents, which explains the difference in the male female ratio and age between the healthy controls and the patients. The gender difference was analyzed and proved relevant for the outcome.

The differences of the populations explain the different results of the analyses in the two studies, but the trend is comparable. The reliability of the scores, expressed as Cronbach's $\alpha$ coefficient was almost identical in the two studies. The relation of the outcome of the CDC Symptom Inventory (DLV) and the SF-36 Physical Functioning score was comparable. The CIS-20 Fatigue and Concentration scores in our analysis were closely related to the CDC Symptom Inventory DLV scores with correlation coefficients that were comparable to the MFI General Fatigue and Mental Fatigue scores in the study of Wagner et al. [6]. 
We limited the number of tests because of clinical relevance for the patients. More tests would have added little to the clinical diagnosis of CFS.

\section{Conclusion}

The Dutch translation of the CDC Symptom Inventory proved to be a reliable tool in the clinical setting of a tertiary care center. The translated version is reliable and the results are comparable to the study of a different population in an English-speaking country. The different response of men and women to the tests warrants further study.

\section{Competing interests}

The author(s) declare that they have no competing interests.

\section{Acknowledgements}

Prof H.R. Scholte for advices and careful reading of the document.

Patients, visitors and co-workers of the CFS and Pain Research Center Amsterdam for their indispensable help.

\section{References}

I. Fukuda K, Straus SE, Hickie I, Sharpe MC, Dobbins JG, Komaroff A, Schluederberg A, Jones JF, Lloyd AR, Wessely S, Gantz NM, Holmes GP, Buchwald D, Abbey S, Rest J, Levy JA, Jolson H, Peterson DL, Vercoulen JHMM: The chronic fatigue syndrome: A comprehensive approach to its definition and study. Annals of Internal Medicine 1994, I 2 I(I 2):953-959.

2. Smets EMA, Garssen B, Bonke B, De Haes JCJM: The Multidimensional Fatigue Inventory (MFI) psychometric qualities of an instrument to assess fatigue. Journal of Psychosomatic Research 1995, 39(3):315-325.

3. Vercoulen JHMM, Alberets M, Bleijenberg G: De Checklist Individual Strength (CIS). Gedragstherapie 1999, 32:3I-36.

4. Reeves WC, Wagner D, Nisenbaum R, Jones JF, Gurbaxani B, Solomon L, Papanicolaou DA, Unger ER, Vernon SD, Heim C: Chronic fatigue syndrome - A clinically empirical approach to its definition and study. BMC Medicine 2005, 3(-):27p.

5. Aaronson NK, Muller M, Cohen PDA, Essink-Bot ML, Fekkes M, Sanderman R, Sprangers MAG, Te Velde A, Verrips E: Translation, validation, and norming of the Dutch language version of the SF-36 Health Survey in community and chronic disease populations. Journal of Clinical Epidemiology 1998, 5 I (I I): I055- 1068.

6. Wagner D, Nisenbaum R, Heim C, Jones JF, Unger ER, Reeves WC: Psychometric properties of the CDC Symptom Inventory for assessment of Chronic Fatigue Syndrome. Population Health Metrics 2005, 3(-):8p.
Publish with Bio Med Central and every scientist can read your work free of charge

"BioMed Central will be the most significant development for disseminating the results of biomedical research in our lifetime. "

Sir Paul Nurse, Cancer Research UK

Your research papers will be:

- available free of charge to the entire biomedical community

- peer reviewed and published immediately upon acceptance

- cited in PubMed and archived on PubMed Central

- yours - you keep the copyright

Submit your manuscript here:

http://www.biomedcentral.com/info/publishing_adv.asp
BioMedcentral 